\title{
Insights from integrating clinical and preclinical studies advance understanding of graft-versus-host disease
}

\author{
Gérard Socié, ${ }^{1,2}$ Leslie S. Kean, ${ }^{3,4}$ Robert Zeiser, ${ }^{5}$ and Bruce R. Blazar ${ }^{6}$ \\ ${ }^{1}$ Hematology-Transplantation, Assistance Publique-Hôpitaux de Paris (APHP), Hospital Saint Louis, Paris, France. ${ }^{2}$ INSERM UMR 976 (Team Insights) and University of Paris, Paris, France. ${ }^{3}$ Division of \\ Pediatric Hematology/Oncology, Boston Children's Hospital, Boston, Massachusetts, USA. DDana-Farber Cancer Institute, Boston, Massachusetts, USA. ${ }^{5}$ Department of Medicine I, Faculty of Medicine, \\ Medical Center-University of Freiburg, Freiburg, Cermany. ${ }^{6}$ Masonic Cancer Center and Department of Pediatrics, Division of Pediatric Blood and Marrow Transplantation \& Cellular Therapy, University of \\ Minnesota, Minneapolis, Minnesota, USA.
}

\begin{abstract}
As a result of impressive increases in our knowledge of rodent and human immunology, the understanding of the pathophysiologic mechanisms underlying graft-versus-host disease (CVHD) has dramatically improved in the past 15 years. Despite improved knowledge, translation to clinical care has not proceeded rapidly, and results from experimental models have been inconsistent in their ability to predict the clinical utility of new therapeutic agents. In parallel, new tools in immunology have allowed in-depth analyses of the human system and have recently been applied in the field of clinical CVHD. Notwithstanding these advances, there is a relative paucity of mechanistic insights into human translational research, and this remains an area of high unmet need. Here we review selected recent advances in both preclinical experimental transplantation and translational human studies, including new insights into human immunology, the microbiome, and regenerative medicine. We focus on the fact that both approaches can interactively improve our understanding of both acute and chronic CVHD biology and open the door to improved therapeutics and successes.
\end{abstract}

\section{Introduction}

Graft-versus-host disease (GVHD) remains the leading cause of nonrelapse death in patients who received allogeneic hematopoietic stem cell transplantation (allo-HSCT) (1). While GVHD classically was clinically divided into acute (early onset, within 3 months after allo-HSCT) and chronic (late onset), these classifications have been refined following the development of the NIH consensus criteria (2). Although the classifications were operational in assigning early signs to acute GVHD and later multiorgan symptomatology to chronic GVHD, the overlap syndrome (3) clearly exemplifies the intersecting and time-independent overlap of these two syndromes. Much of our previous understanding of acute GVHD pathophysiology was gained from experimental models (reviewed in refs. 4-6), while some information relevant to the disease's mechanisms was derived from humans (reviewed in ref. 7). Clinicians have long viewed the goal of trans-

Conflict of interest: GS serves on the advisory board and speaker bureau for Novartis, Incyte, Elsalys, and Regeneron Pharmaceuticals and on the speaker bureau for Amgen, and has received a research grant from Alexion. LK serves on the scientific advisory board for HiFiBio, and receives research funding from Kymab Ltd., Magenta Therapeutics, BlueBird Bio, and Regeneron Pharmaceuticals; consulting fees from Equillium, FortySeven Inc., Novartis Inc., EMD Serono, Gilead Sciences, and Takeda Pharmaceuticals; and grants and personal fees from Bristol Myers Squibb. RZ receives speaker fees from Novartis, Incyte, and Mallinckrodt. BRB serves as an advisor to Magenta Therapeutics and BlueRock Therapeutics; receives research funding from BlueRock Therapeutics, Rheos Medicines, and Carisma Therapeutics; and is a cofounder of Tmunity Therapeutics.

Copyright: @ 2021, American Society for Clinical Investigation.

Reference information: / Clin Invest. 2021;131(12):e149296.

https://doi.org/10.1172/JCl149296. lating experimental results to therapeutic strategies as being out of reach or prone to failure, while laboratory scientists have perceived clinical GVHD research as non-mechanistic. Here, rather than extensively reviewing the previous literature (see refs. 4-6 for this purpose), we aim to highlight how, recently, studies of both human immunology and experimental transplantation have worked synergistically to advance the field in GVHD pathophysiology, diagnosis, and treatment.

\section{Studies of alloreactivity identify potential therapeutic GVHD targets}

When considering the most impactful strategies to study alloreactivity, we include animal models, in vitro studies, and the interrogation of human samples. The most tenable animal models have used inbred strains of mice, in which the many variables contributing to alloreactivity can be controlled. These include (a) degree of MHC disparity; (b) multiple variations in the intensity of pretransplant conditioning, bone marrow, and T cell doses; (c) clinical parameters (age and body weight); (d) genetic manipulation of specific cell populations; (e) use of inhibitors of key molecular contributors to alloreactivity; and (f) control of the environment (e.g., specific pathogen-free or microbiome alterations). Both canine and nonhuman primate (NHP) GVHD models (8-12) have also been established, and what they lack in genetic flexibility, they gain in their closer evolutionary proximity to humans and the ability to rigorously evaluate clinical strategies in a highly translational setting. This is perhaps most true of NHPs, given that many novel targeted therapeutics developed for patients do not cross-react with mouse or canine targets (13-15). 
While preclinical in vivo models may be the most tractable to discern mechanisms and strategies to control alloreactivity, a major advance has been the deepening interrogation of human alloreactivity through flow cytometry and molecular studies. These studies have included detailed multiparameter flow cytometry, mass cytometry, and RNA-Seq analysis of patients undergoing allo-HSCT, most studying the blood, but with an ever-increasing interrogation of GVHD target tissues. While more low-throughput techniques have nominated several cell types and proteins as being associated with GVHD, the expanding use of transcriptomics has increasingly linked immune pathways and gene expression networks to GVHD (14-18). This includes the identification of transcriptomic profiles associated with acute GVHD (19), and of the first CD4 ${ }^{+} \mathrm{T}$ cell transcriptomic network associated with acute GVHD severity (20). A method of tracking human alloimmune responses through mixed-lymphocyte reactions and subsequent tracking of clones in vivo after organ transplantation deserves special mention (20-22). When combined with single-cell transcriptomic methods, this method could be an especially powerful tool for studying the pathologic activation networks in human GVHD.

\section{Improving tools will catalyze translational progress for GVHD}

Our expanding insights into the human immune responses driving GVHD are closely linked with the technical advances that enable us to measure the behavior of genes, mRNA (including single-cell transcriptomics), proteins (proteomics), metabolites (metabolomics), cells (mass cytometry), and epigenetic modifications, many of which have been accomplished in human immunology on the basis of studies on vaccination, cancer, and viral infections. More generally, we endorse Mark Davis's view $(23,24)$ that studying the immune system in humans offers a direct link to medicine (i.e., "translation"), but that profiling immunity in humans is only the first step; biological validation studies in animal models (or human organoids) represent essential next steps to close the loop from humans, to mice, to translational medicine, including GVHD.

\section{From basic immunology and experimental data to pathophysiology}

Studies conducted mostly in mice have demonstrated that pretransplant conditioning and early posttransplant responses elicit both danger-associated molecular patterns and pathogen-associated molecular patterns. These studies have shown that pretransplant irradiation results in increased expression of microbiota-derived TLR ligands, which triggers macrophage IL-12/IL-23 secretion and innate lymphoid cell type 1 (ILC1) and conventional T cell (Tconv) IFN- $\gamma$ release $(6,25)$. Limited lower gastrointestinal (GI) microbiota diversity due to antibiotic use has been associated with increased GVHD (26), whereas changes in the pulmonary microbiome have been associated with bronchiolitis obliterans (27), pointing to microorganisms as initiating and/or amplifying disease pathogenesis. Relevant to GI GVHD, intestinal macrophages stimulate dendritic cells to release IL-12, which results in the secretion of IFN- $\gamma$ via stimulation of host ILC1 and host $\mathrm{T}$ cells and differentiation of naive donor $\mathrm{T}$ cells into Th1/ type $1 \mathrm{CD}^{+} \mathrm{T}(\mathrm{Tc} 1)$ cells and Th17/IL-17-secreting cytotoxic T (Tc17) cells (28). GI injury has been attributed to donor perforinand granzyme-mediated T cell cytolysis (reviewed in refs. 4, 6). Recent studies have elucidated even more precisely the mechanism(s) of GI injury: Using 3D imaging and ex vivo intestinal organoid cultures, intestinal stem cells (ISCs) were identified as the primary GVHD target of invading donor T cells $(29,30)$. ISC injury then occurs as a result of IFN- $\gamma$-induced JAK1/STAT1 proapoptotic gene expression.

IFN- $\gamma$ also drives MHC class II upregulation on antigen-presenting cells (APCs) and intestinal epithelial cells (IECs) in the ileum and proliferation of $\mathrm{CD}^{+} \mathrm{T}$ cells (25). Similarly, ex vivo murine and human organoid cultures exposed to IF $\mathrm{N}-\gamma$ upregulated MHC class II expression (29-31), revealing a novel mechanism for initiating $\mathrm{T}$ cell-mediated lethal GVHD involving an IFN- $\gamma$ axis and centering on ileal ISC MHC class II expression. Collectively, these findings support testing strategies that impair donor $\mathrm{T}$ cell homing to the crypt or prevent IFN- $\gamma$-mediated ISC cell death. A randomized phase III trial of the JAK1/2 inhibitor ruxolitinib in patients with steroid-refractory (SR) acute GVHD was successful (32). As IFN- $\gamma$ activates JAK1/2, ruxolitinib' s beneficial effects may (at least in part) include reduced IL-12/IL-23 production, IFN- $\gamma$-induced ISC apoptosis, and MHC class II upregulation. Understanding of this trajectory (from IFN- $\gamma$ to JAK1/2 signaling to subsequent augmentation of $\mathrm{T}$ cell pathogenicity) is an instructive example of how detailed interrogation of key $\mathrm{T}$ cell pathways can catalyze therapeutic development, and how clinical trials linked to these novel therapeutics can, in turn, deepen our mechanistic understanding of GVHD.

\section{JAK inhibition}

JAK1/2 inhibition was tested in different acute GVHD models $(33,34)$, and functional analyses showed that JAK1/2 inhibition reduced MHC class II expression on APCs (35) and neutrophil migration (36). Based on results of a limited number of patients who had failed multiple therapies for acute GVHD (37), prospective trials were initiated using ruxolitinib. A single-arm phase II study (REACH-1) confirmed ruxolitinib's activity in the setting of SR acute GVHD (38), and a multicenter randomized phase III trial (REACH-2) formally tested ruxolitinib's superiority to best available therapy (BAT) (32). The primary study objective (overall response at day 28) was met, with significantly better response with ruxolitinib than with BAT (62.3\% vs. $39.4 \%)$. Finally, a third trial (REACH-3) in patients with SR chronic GVHD also demonstrated a significant response rate over BAT (39). Based on promising activity in preclinical models (40) and in a phase I trial (41), itacitinib (a selective JAK1 inhibitor) was tested in a randomized phase III first-line treatment trial in acute GVHD. Although the trial did not reach the primary endpoint for response rate, the complete response rate was significantly higher in patients with high-risk acute GVHD (42). The rather rapid clinical translation of JAK-targeted therapeutics for GVHD is a notable example of a new era of deep mechanistic insights into this disease; driven by decades of preclinical work, these insights have now caught the attention of relevant pharmaceutical companies to successfully develop, market, and repurpose therapeutics for GVHD control. 


\section{Costimulation}

The central role of $\mathrm{T}$ cell costimulation in GVHD was established as early as 1994, when CD28:CD80/86 costimulation blockade with CTLA-4-Ig was demonstrated to significantly prevent GVHD and improve survival in a mouse GVHD model (43). Canine studies recapitulated these results (44), but it was not until supportive NHP studies were completed $(13,15)$ that translation of this strategy for clinical GVHD prevention occurred. Since the initial report of the abatacept formulation of CTLA-4-Ig for clinical GVHD prevention in 2013 (45), there have been increasing reports of its impact on both acute GVHD prevention and chronic GVHD treatment. In the largest trial to date, abatacept improved acute GVHD-related outcomes after unrelated-donor transplantation, an observation that led to a breakthrough therapy designation for this drug (20).

Although CD28:CD80/86 signaling serves as the canonical costimulation pathway in GVHD, mouse and NHP studies have identified other second signals, which, when interrupted, can potently restrain alloreactivity. Many represent targets for which available clinical therapies exist and are primed for investigation in GVHD. Among the most noteworthy targets are the TNF receptor and immunoglobulin superfamily members CD40 ligand (CD154; refs. 46-48), OX40L (CD252; ref. 49), and ICOS (inducible T cell costimulator, CD278; ref. 50), as well as Notch signaling pathways $(13,43,47,48,51)$. CD154 blockade has been demonstrated in mouse and NHP models of alloreactivity to be a potentially high-value target for preventing effector $\mathrm{T}$ cell activation while preserving regulatory T cell (Treg) signaling. Although original anti-human CD154 antibody formulations were associated with thromboembolic complications, new Fc-silent formulations were not and are moving to the clinic. Anti-CD154 antibodies have not yet been tested clinically for GVHD prevention or treatment; their unique attributes suggest that these trials should be strongly considered $(48,51)$.

A major issue facing costimulation blockade-based strategies for GVHD control is controlling costimulation-induced effector T cell activation while retaining Treg function and homeostasis (14, 15). Recent work has identified blockade of OX4O/OX4OL costimulation as a particularly compelling strategy for GVHD control. When combined with mTOR inhibition (sirolimus) in the NHP model of GVHD, OX4OL blockade effectively controlled effector $\mathrm{T}$ cell activation while supporting Treg reconstitution, leading to effective GVHD prevention (14). This anti-OX4OL antibody used in NHP studies is currently in clinical trials for autoimmune diseases and poised for translation to the clinic for GVHD.

In contrast to the constitutive expression of many costimulation molecules, ICOS has low baseline expression but is upregulated within a few days after T cell activation. Foundational studies determined that ICOS and CD28 synergistically promote GVHD (52), whereas checkpoint signaling through CTLA-4 is required for T cell tolerance regardless of ICOS signaling (53). These results suggest that inhibition of ICOS, along with selective blocking of CD28 (through anti-CD28 antibodies, rather than CTLA-4-Ig), may represent a promising approach for GVHD control.

While prolonged (rather than transitory) blockade of multiple costimulation pathways has been necessary to improve GVHD outcomes (reviewed in ref. 54), targeting of the Notch pathway in mice has been notable as a monotherapy even with very shortcourse pathway inhibition. Notch signals delivered during the first 48 hours after transplantation drive Tconv proinflammatory cytokine production and inhibit Treg expansion (55-57). Because of this unique mechanism of action, short-term Notch inhibition induces long-term GVHD protection in mice. The ability to modulate effector $\mathrm{T}$ cell priming and downstream activation with a time-limited inhibition of Notch signaling suggests that Notch blockade could play a salutary role in clinical GVHD.

The above examples offer a compelling illustration of the power of preclinical studies focusing on GVHD biology and mechanisms that facilitate the pathway to clinical translation.

\section{Microbiome and metabolism}

In recent years, microbiome research has greatly expanded as a result of technical advances in sequencing-based analysis of microbial flora of the human intestine that have allowed better understanding of the microbiome's interaction with the host immune system after allo-HSCT. Independent work has shown that the commensal microbiome is frequently dysregulated and reduced in its diversity following allo-HSCT and that this dysbiosis is connected to acute intestinal GVHD (58-61). Certain bacteria, such as butyrate-producing Blautia, were associated with a better outcome of patients developing acute GVHD (62), while others, such as enterococci, were identified as an acute GVHD risk factor (63). Microbiome-derived metabolites that modulate IEC damage, including butyrate, provide a connection between microbiome changes and development of acute intestinal GVHD (64). Reduced butyrate production led to increased acute GVHD severity (64). This finding has important clinical implications, as an increased GVHD-related mortality was observed in patients treated with certain broad-spectrum antibiotics (65). Dietary measures may also impact outcome, as dietary lactose depletion attenuated Enterococcus outgrowth, reducing acute GVHD in experimental models (63). Additionally, GVHD amelioration was made possible by immunization against the conserved microbial surface poly-N-acetylglucosamine, which depleted bacteria penetrating the intestinal wall, thereby reducing neutrophil activation while sparing microbial diversity $(36,66)$. Diversity of not only bacteria (bacteriome) but also the virome (67) and mycobiome (68) was connected to intestinal GVHD. Fecal microbiota transplants (FMTs) have been evaluated as therapy in patients suffering from SR acute GVHD (69). Other studies (yet involving few patients) analyzed the impact of FMT as GVHD prophylaxis or in patients with SR GVHD in patients $(70,71)$. However, FMT is still considered an experimental approach and needs validation and safety data in carefully designed prospective randomized clinical trials.

A major goal in reducing acute GVHD incidence and severity is avoiding transmigration of intestinal bacteria through the damaged intestinal wall, as this can lead to the recruitment and activation of neutrophils that trigger GVHD by direct tissue damage (72) and by antigen presentation to donor T cells (36). Amphiregulin (AREG), an EGFR ligand, participates in the intestinal epithelium repair during GVHD in mice (73), and high circulating AREG levels, indicative of intestinal injury, were associated with an unfavorable outcome in patients with acute GVHD (74). 
Microbiome-associated metabolic alterations using highthroughput metabolomics in allo-HSCT recipients were linked to acute GVHD in two cohorts of genotypically HLA-identical related recipient and donor pairs (75). At acute GVHD onset, a significant variation in microbiota-derived metabolites occurred - in particular, aryl hydrocarbon receptor ligands, bile acids, and plasmalogens (75). The role of bile acids in acute GVHD was further supported by functional studies indicating that tauroursodeoxycholic acid treatment reduced GVHD by decreasing MHC expression on APCs and decreasing intestinal apoptosis (76). In other studies, metabolic reprogramming in $\mathrm{T}$ cells impacted both GVHD (77) and graft-versus-leukemia (GVL) effects (78). Pathogenic $\mathrm{CD}^{+} \mathrm{T}$ cells were found to be highly glycolytic by transcriptomic, protein, and metabolomic analyses and could be detected in early acute GVHD by noninvasive metabolic imaging (79). Lysosomal acid lipase (LAL) mediates the intrinsic lipolysis of cells to generate free fatty acids; pharmacological targeting of LAL reduced experimental GVHD (80). In mouse models, donor $\mathrm{T}$ cells undergo metabolic reprogramming after allo-HSCT, switching from fatty acid $\beta$-oxidation and pyruvate oxidation via the tricarboxylic acid cycle to aerobic glycolysis, thereby increasing dependence on glutaminolysis and the pentose phosphate pathway (81). Conversely, inhibiting glycolysis by targeting mTORC1 reduced GVHD severity (81).

While our understanding of the complex intersections between the microbiome and posttransplant immune pathology has deepened in the past decade, the new insights have not yet been broadly or effectively translated to the clinic but represent one of the major opportunities, and hurdles to overcome, in clinical development of GVHD.

\section{Regenerative approaches}

Tissue-intrinsic and -extrinsic alloreactive immune cell-independent, parenchymal tissue-specific mechanisms exist that can mitigate damage of host tissues despite pathologic acute GVHD; these mechanisms are known as tissue tolerance (82). In contrast to approaches that seek to restore immune system balance, strategies that protect and speed the regeneration of tissues from injury have emerged, especially with respect to GI GVHD. Again, preclinical studies have identified several therapeutic approaches aimed at promoting intestinal regeneration following immune destruction, including keratinocyte growth factor (KGF), IL-22, R-spondin 1 (R-Spo1), and glucagon-like peptide-2 (GLP-2).

KGF (also known as FGF-7), which stimulates epithelial cell proliferation in GI tract in both mice and humans, significantly reduced GVHD pathology and improved survival when given before conditioning (83). This pretransplant-only regimen provided proof of principle that direct targeting of tissue injury and repair could impact posttransplant outcome. In contrast to positive results seen in a phase III study of autologous recipients receiving a highly mucolytic conditioning regimen and peripheral blood stem cells (84), a phase I/II randomized placebo-controlled trial (85) showed that while severity of oral mucositis could be reduced by palifermin (KGF), GVHD was unaffected. More promising results are hoped for in clinical trials targeting IL-22, a critical regulator of epithelial homeostasis (86). In the context of inflammatory bowel disease and GVHD, IL-22, which has both antiinflammatory and proinflammatory effects, was reported to directly enhance ISC protection and regeneration (87). A clinical trial using IL-22 IgG2-Fc (F-652) to provide IL-22 signals to the host for treatment of lower GI GVHD has finished recruitment (ClinicalTrials.gov NCT02406651), and results are eagerly anticipated.

Wnt signaling regulates IEC proliferation. In a mechanism dependent on repair from conditioning regimen injury, R-Spo1, a potent canonical Wnt/ $\beta$-catenin signaling agonist, protected against ISC damage, enhanced restoration of injured intestinal epithelium, and inhibited inflammatory cytokine cascades in a GVHD model (88). R-Spo1 was also used to restore the intestinal microbial equilibrium: it was shown to stimulate ISC differentiation into Paneth cells, which then promoted luminal secretion of $\alpha$-defensins that are active against many Gram-negative and Gram-positive bacteria, fungi, and enveloped viruses (89).

The final GI-regenerative approach we will highlight focuses on GLP-2, an enteroendocrine tissue hormone secreted by intestinal L cells found predominantly in the ileum and colon. A recent study reported that acute GVHD reduced intestinal GLP2 levels in mice and patients developing GVHD (90). In a therapeutic approach, GLP-2 agonist (teduglutide) treatment reduced de novo acute GVHD and SR GVHD, without compromising GVL effects. Exogenous GLP-2 enhanced regeneration of Paneth cells and ISCs, which then increased antimicrobial peptide production and caused microbiome modifications. Additionally, GLP-2 expanded intestinal organoids and reduced expression of apoptosis-related genes in intestinal cells (90). Besides these results derived from studies in mice, low numbers of $\mathrm{L}$ cells in intestinal biopsies and high serum levels of GLP-2 were associated with higher incidence of nonrelapse mortality in patients undergoing allo-HSCT. These observations suggest that GLP-2 could be a high-impact pathway to further clinically develop, especially given its promising safety/efficacy balance.

\section{Pathogenic resident memory T cells in GVHD}

One of the major challenges that the field must face is that $\mathrm{T}$ cells, key contributors to tissue destruction during GVHD, are also critical for reconstitution of antipathogen and antitumor immune surveillance. This is especially true of resident memory T cells (Trms), which have been demonstrated to be essential for tissue-specific antipathogen immunity, both before and after transplant (reviewed in refs. 91, 92). Several recent studies have explored the immunology of the pathogenic Trms that are associated with tissue-specific GVHD lesions, with some surprising observations emerging. Indeed, even before the formal description of Trms, a population of tissue-destructive, TGF- $\beta$ dependent, $\mathrm{CD} \mathrm{O3}^{+} \mathrm{CD}^{+} \mathrm{T}$ cells was described as critical for GVHD-associated GI destruction in mice (93), and recent work has demonstrated long dwell times of both $\mathrm{CD}^{+}$and $\mathrm{CD} 8^{+} \mathrm{T}$ cells in the skin in a mouse model of cutaneous GVHD (94). In NHPs, serial infusions of fluorescently tagged anti-CD 45 mAbs coupled with single-cell RNA-Seq have recently enabled a spatial and temporal exploration of the evolution and pathogenesis of donor $\mathrm{CD}^{+}$Trms in the GI tract during acute GVHD (18). These studies demonstrated that while T cells in the act of infiltrating the GI tract do not yet express the canonical transcriptomic and protein 
A

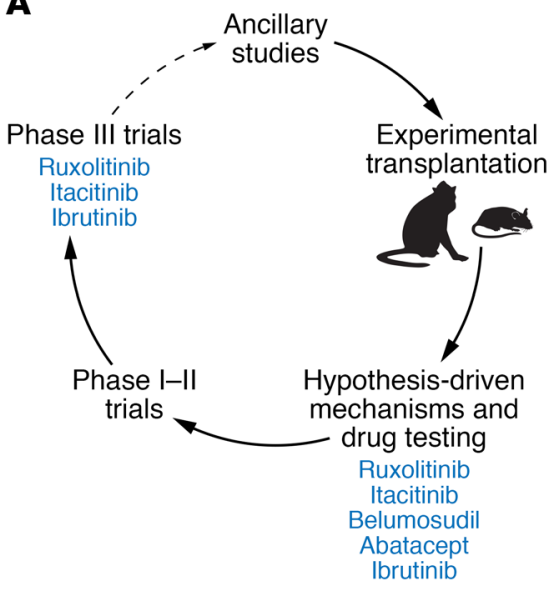

B

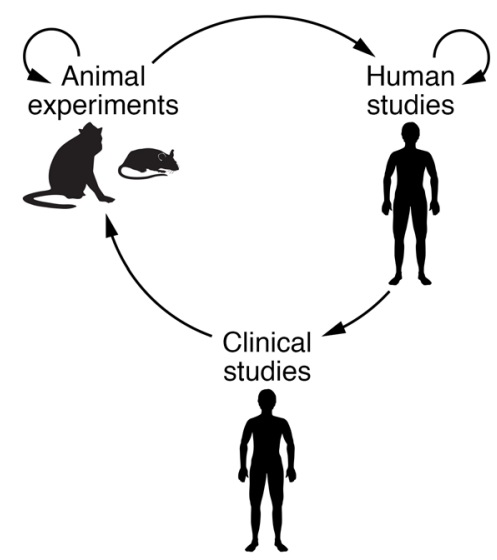

Figure 1. Comparing current and proposed schema for drug development in GVHD. (A) Current schema for drug development in GVHD. As of today, new therapeutics are mostly based on scientific findings derived from experimental models that either test a drug or use a knockout system to assess the role of a given pathway in GVHD. These experimental results trigger the development of phase I and II clinical trials and eventually lead to the conduction of randomized trials. Example candidates resulting from such a process are depicted. It is of note that very few ancillary studies coming from clinical trials lead to further mechanistic studies in experimental models. (B) We proposed a shift in the paradigm of drug development in GVHD treatment wherein clinical development can either come from animal models or are derived from human samples. In all cases, results from clinical trials can generate novel hypothesis that need to be tested experimentally.

expression signature of Trms, invading $\mathrm{T}$ cells rapidly acquire a Trm profile associated with their intestinal residency. However, the existence of these cells is likely distinct from the protective Trms that survey and defend organs from infection. Donor Trms in the NHP GI tract exhibit both flow cytometric and transcriptomic signs of pathogenicity including increased proliferative and cytotoxic capacity compared with both Trms from healthy controls and, importantly, residual host Trms in the GI tract after HSCT. These data inform a heuristic that is central to the developing molecular atlas of GVHD: that cell types and cell states that contribute to protective immunity during homeostasis can evolve and differentiate into pathogenic subtypes, able to induce significant tissue damage during alloreactivity. While in the NHP model of acute GVHD, donor T cells in the intestine are more pathogenic than residual host $\mathrm{T}$ cells, recent data suggest that for some tissues (skin in particular), host cells are not only longlived but can become pathogenic after HSCT. This was recently demonstrated by two independent studies $(95,96)$, both exploring the role of host $\mathrm{T}$ cells in human GVHD. They demonstrate that these host cells can survive for more than a decade after HSCT, are highly proliferative, and produce cytokines (including both IFN- $\gamma$ and IL-17). The retention of host Trms in GVHD target organs in patients, and their ability to evolve toward pathogenic cell states, underscore the complexity of pathogenic networks driving GVHD. It warns us that even cell types that normally demonstrate homeostatic and protective functions can evolve toward pathogenicity in the highly inflammatory environment of post-HSCT GVHD. This double-edged sword of T cell immunity after allo-HSCT underscores the need to perform detailed studies of human GVHD, as no model system can fully recapitulate the complex immune networks at play in patients.

\section{Posttransplant cyclophosphamide}

The complexity of clinical approaches to GVHD control is well encapsulated by the translation of posttransplant cyclophosphamide (PTCy) to the clinic, a major advance in the last decade of GVHD prophylaxis. Administration of high-dose PTCy following allo-HSCT has dramatically changed the therapeutic landscape, allowing the wide use of haploidentical donors, and is currently increasingly used for HSCT from unrelated donors $(97,98)$. However, the mechanistic underpinnings of PTCy efficacy are still not fully understood. Early data on skin allograft rejection suggested that PTCy selectively eliminated alloreactive $\mathrm{CD}^{+}$cells (reviewed in ref. 97). Lately, much attention has been paid to dissection of the GVHD-protective role of PTCy (99), challenging previous paradigms that alloreactive $\mathrm{T}$ cell elimination and thymic clonal deletion are primary mediators of PTCy efficacy. More recently, studies have indicated that PTCy causes alloreactive donor $\mathrm{T}$ cell dysfunction, rather than elimination, and promotes the rapid, preferential recovery of highly suppressive $\mathrm{FoxP}^{+} \mathrm{CD}^{+}$Tregs that prevents the generation of new donor GVHD-causing T cells $(100,101)$. This dysfunction combined with Treg reconstitution has led to effective control of chronic GVHD with PTCy, but leaves transplant patients open to some unwanted outcomes, such as the risk of relapse and delayed immune reconstitution. These issues again highlight the importance of continuing detailed mechanistic immune studies on patients undergoing transplant with PTCy to identify predictors of success or toxicity with this approach and optimize its use in HSCT.

\section{Chronic GVHD}

Thus far, this Review has focused predominantly on acute GVHD; here we will discuss selected developments in the elucidation of chronic GVHD pathophysiology that were reported after the publication of a series of comprehensive reviews in 2017 (5, 102-104). In contradistinction to the cellular inflammatory, destructive process of acute GVHD, chronic GVHD is an autoimmune-like disorder, typified by fibrosis and often associated with antiinflammatory Th2 cells, macrophage infiltration, pathogenic B cells, and allo- and autoantibodies.

Thymic injury. Thymic cell injury from chronic GVHD results in loss of thymic epithelial cells (TECs). Additionally, the autoimmune regulatory (AIRE) protein, responsible for transcription and display of tissue-specific antigens in the thymus, is dysfunctional. AIRE dysfunction coupled with reduction of medullary TECs results in a failure of deletion of autoreactive T cells, allowing their escape into the periphery, and in thymus-derived Treg generation. In concert with thymus-derived Tregs, regulatory invariant natural killer (iNK) cells were noted to be deficient in mouse chronic GVHD models $(105,106)$. Treatment of established chronic 


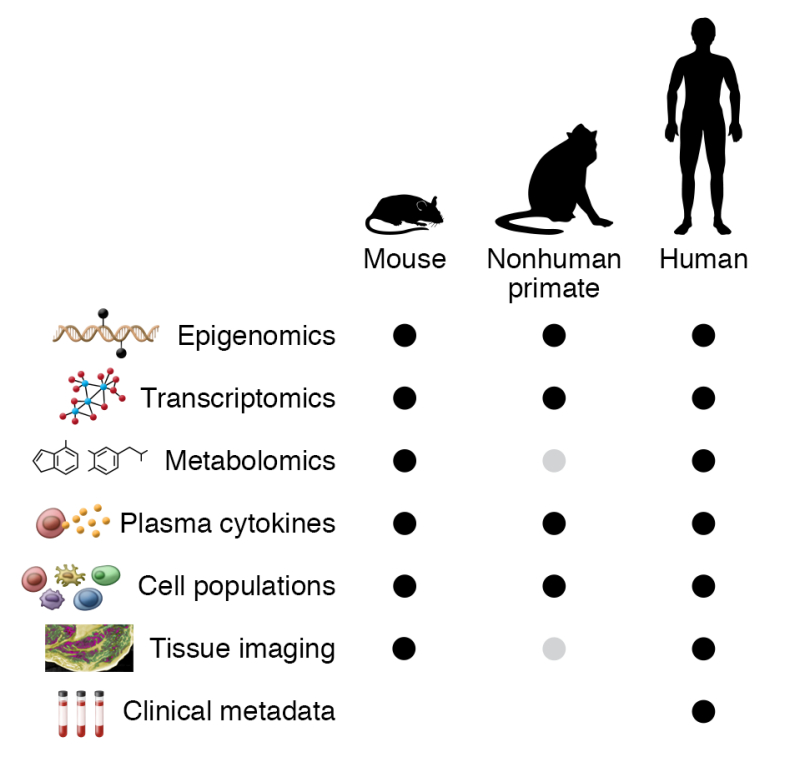

Figure 2. Biological tools for drug development in GVHD. The figure shows various biological tools that can be used either in experimental transplantation or in human samples to generate data suggestive of therapeutic interventions to trigger a clinical trial. Gray circles indicate that the technique has not yet been widely used in the indicated model.

GVHD with $\alpha$-galactosylceramide to stimulate iNK cell production or iNK-T cell infusion reversed chronic GVHD manifestations in an IL-4- and Treg-dependent fashion. Moreover, loss of ILC3s that signal TECs for repair impairs thymopoiesis and immune recovery (104). Finally, damage to lymph node nonhematopoietic cells, especially host fibroblastic reticular cells (FRCs) shown to be depleted by radiation conditioning, continues the failed process of peripheral display of tissue-restricted antigens (107). With the paucity of thymus-derived Tregs, autoreactive T cells are inadequately controlled and chronic GVHD can ensue. Additionally, FRCs that express Delta-like Notch ligands, which bind Notch receptors on donor $\mathrm{T}$ cells and prime alloreactivity, are depleted (57). Notch pathway genetic deficiency or antibody blockade can thereby also abrogate chronic GVHD, by reducing the priming of alloimmunity (57), decreasing donor T cell cytokine secretion, and increasing Tregs (56).

Fibrosis. Deposition of allo- and autoantibody in chronic GVHD organs can cause tissue fibrosis. Preclinical data are consistent with chemokine-mediated macrophage recruitment into chronic GVHD organs, Fc receptor engagement, and elaboration and secretion of profibrogenic factors $(5,102)$. Interestingly, sclerodermal fibroblasts can upregulate CD47 that hinders macrophage removal of diseased cells (108). Pirfenidone [5-methyl-1-phenyl-2-(1H)-pyridone] is an antifibrotic SMAD2/3 inhibitor that decreases hydroxyproline, fibrosis, procollagen I and II, PDGF isoforms, TGF- $\beta$, and FGF, while increasing reactive oxygen species scavenging (105). Pirfenidone decreased macrophage migration in response to monocyte chemoattractant protein- 1 and IL-17A, resulting in markedly reduced fibrosis in mice with active chronic GVHD. Mice and patients with chronic GVHD have an increased frequency of IL-17-polarized T cells; in mice, treatment with a small-molecule ROR $\gamma$ t inhibitor or neutralizing IL-17 anti- body could ameliorate disease (109). Selective Rho-associated kinase 2 (ROCK2) inhibition decreases IL-21 secretion needed for germinal center (GC) responses and blunts constitutively upregulated phospho-STAT3 in splenocytes, reducing IL-17 secretion and fibrosis. Additionally, the selective ROCK2 inhibitor KD025 hinders cell motility and actin stress fiber formation that can have a direct antifibrotic effect. KD025 was able to reverse or diminish the progression of chronic GVHD (110).

Pathogenic B cells. Depending on the exact conditions of murine chronic GVHD models, the epicenter of disease initiation has been attributed to increased number and size of GCs, wherein somatic hypermutation and selection of high-affinity antibody-producing GC B cells occur $(6,103)$. A small-molecule BCL6 inhibitor and BCL6 deficiency in either donor T cells or B cells were required for GC formation and chronic GVHD (111). CD4 ${ }^{+}$T cells expressed C5a receptor 1 (C5aR1). C5aR1 signaling was needed to amplify IL-6-dependent c-MAF and IL-21 expression by phosphorylating phosphokinase B (AKT) and activating the mammalian target of rapamycin (mTOR). These data suggest that C5aR1 may be a useful therapeutic target for prevention and/or treatment of individuals with T follicular helper cell-dependent chronic GVHD (112). The REACH-3 trial of ruxolitinib in SR chronic GVHD demonstrated a significant response rate over BAT (39). As IL-6 drives $\mathrm{B}$ cell proliferation and is critical for plasma cell differentiation and antibody production, the efficacy of ruxolitinib may be due, at least in part, to lowering of IL- 6 secretion.

$T$ and $B$ cell cooperation. Other studies performed in different strain combinations observed GC depletion. Alternatively, autoand alloantibody production may occur as a result of $\mathrm{CD} 4^{+}$pre-T follicular helper cells' cooperativity with pre-GC B cells in extrafollicular areas within secondary lymphoid organs. Here, BCL6 expression was dispensable for chronic GVHD due to GC but not extrafollicular T/B cooperativity. Most recently, a nonlymphoid location of tissue-resident $\mathrm{ICOS}^{+}$, IL-21-producing PSGL $1^{10} \mathrm{PD}-1^{+}$ $\mathrm{CD}^{+} \mathrm{T}$ cells with properties of $\mathrm{B}$ cell helper cells was shown to augment support of PD-L2-expressing B cell differentiation into plasma cells and subsequent autoantibody production (113). Consistent with nonlymphoid localization data, infiltration of chronic GVHD target organs (lung, liver) by $\mathrm{CD}^{+} \mathrm{T}$ cells and colocalized $\mathrm{B}$ cells was also reported in the GC-dependent model (114). Whether there is a single modality or multiple modalities that led to antibody deposition in humans is unknown.

Common to each of the aforementioned mechanisms is the engagement of $\mathrm{T}$ helper cells that support $\mathrm{B}$ cell maturation, antibody production, and subsequent plasma cell generation. In a mouse model, B cell lymphopoiesis in the bone marrow and B cell immune recovery were impeded by the known Treg deficiency in chronic GVHD that resulted in failure to control Tconv inhibition of B cell lymphopoiesis (115). Nonetheless, B cell lymphopenia does not mitigate antibody-induced chronic GVHD pathogenesis, as chronic GVHD mice and patients have a lowered B cell receptor (BCR) threshold and B cell hyperresponsiveness (116, 117). Additional pathways essential for chronic GVHD in some mouse models and patients include splenic tyrosine kinase (Syk) $(118,119)$; Bruton's tyrosine kinase (BTK) and IL-2-inducible T cell tyrosine kinase (ITK) (120); PI3K $\delta$ (121); and inositol 1,4,5-trisphosphate 3-kinase B (ITKPB) (122), which phosphorylates ino- 


\section{Table 1. Strengths and weaknesses of experimental and clinical approaches to studying GVHD}

\begin{tabular}{|c|c|}
\hline & Strengths \\
\hline Experimental models & $\begin{array}{l}\text { - Reproducibility } \\
\text { - Ability to target minor, major, or haplo- antigens } \\
\text { - New tools: inducible knockout, knockin, gene editing (CRISPR) } \\
\text { - In-depth analyses of GVHD target tissues (using new technologies) } \\
\text { - Allow testing of new drugs and antibodies }\end{array}$ \\
\hline Phase I/II clinical trials & $\begin{array}{l}\text { - Describe mainly toxicity; first evidence for efficacy } \\
\text { - Could be used to develop ancillary studies using omics and single-cell technologies } \\
\text { for detailed mechanistic studies in peripheral blood, and with even the smallest } \\
\text { tissue biopsies } \\
\text { - Could be used to design biomarker-based intervention }\end{array}$ \\
\hline Phase III clinical trials & $\begin{array}{l}\text { - Gold standard for new drug indication } \\
\text { - Goal: inclusion of biological and mechanistic endpoints, to get the most } \\
\text { "bang for the buck" from these expensive clinical studies }\end{array}$ \\
\hline Comments & $\begin{array}{l}\text { Failure of trials to meet statistically defined endpoints may be due to either } \\
\text { clinical trial design or preclinical modeling, which may have failed to predict } \\
\text { the optimal trial design. Many trials are designed for which the difference } \\
\text { between } 2 \text { treatment arms is predicted to be fairly small ( } 10 \%-15 \%) \text {. In this } \\
\text { situation, when the expected endpoint is not reached, it could be because } \\
\text { of a real clinical failure of the new agent or, equally likely, could be due to an } \\
\text { overperforming control cohort or an underpowered study design. } \\
\text { The promise and hope is that the use of omics and single-cell technologies to } \\
\text { perform detailed mechanistic studies with patient peripheral blood samples } \\
\text { and, even more importantly, with tissue biopsies will improve the biological } \\
\text { insights, and hence the predictive power of clinical trials. }\end{array}$ \\
\hline
\end{tabular}

Weaknesses

- Age limitations: almost exclusively young mice Inbred (mice)

- Rarely use GVHD prophylaxis

- Conditioning: almost exclusively irradiation-based

- Efficacy bias inherent to study design

- Patient selection affects outcomes

- Use of historical controls as evidence of efficacy

- The net benefit of any drug could be underestimated or overestimated

- Almost never used for biological studies

- Can fail in identifying the correct risk categories of patients to enrol

- Risk of failure to identify clinical differences between control and experimental groups, even if a biological effect is present

Clinical trials, until now, mainly failed to include biological and mechanistic studies when investigating the clinical impact of new GVHD therapeutics.

With increasing depth of and range in omics-type data, there is a strong need for standardization of some biological parameters. sitol trisphosphate (IP3) to negatively regulate calcium flux. Syk, BTK/ITK, and PI3K $\delta$ have been translated into the clinic and generated responses in SR chronic GVHD. B cells from chronic GVHD patients were found to be activated and primed for survival via B cell-activating factor (BAFF) (123). High BAFF/B cell ratios have been reported in patients recovering from myeloablation and are associated with active chronic GVHD (124), as high BAFF levels elevate BCR responses (117).

In aggregate, these data suggest that multiple pathways are involved in chronic GVHD pathogenesis, maintenance, and fibrosis generation. Strategies (proteins, antibodies, pharmacologic agents, cells) that target multiple pathways may have a higher likelihood of responses across a broad population of chronic GVHD with distinct manifestations.

\section{Deciphering human immunology of GVHD}

The uses of omics in deciphering mechanisms of GVHD in patients, mice, or NHPs are still limited but are highly likely to increase substantively in the coming years. Use of high-throughput metabolomics allowed a detailed description of the gut microbiota's influence on the plasma metabolome (75), and new studies in humans (19) and NHP (18) have described the activated transcriptome at the onset of acute GVHD. Somewhat surprisingly, very few studies have used mass cytometry to decipher the different T cell subsets (125) at GVHD onset or their therapy-induced modifications, although several studies are ongoing.
As reviewed above, most recently, two research groups focused on the role of Trms in acute GVHD in humans $(95,96)$. One group has shown that donor-derived cells with allogeneic stimulatory capacity infiltrate GVHD skin and may mediate direct pathogenicity as well as indirect enhanced allostimulatory effects on host Trms (126). These newest studies not only highlighted the unforeseen role of Trms in acute GVHD but also underscored the unforeseen origin of Trms - a sizeable proportion being of recipient origin - that may undergo allogeneic stimulation by infiltrating activated donor monocyte-derived macrophages. These results not only pointed out the critical need to move from blood- to tissue-based analyses in human GVHD, but also pointed to an evolution in the prevailing mechanistic paradigm for GVHD, by suggesting a significant contribution of host cells in the pathophysiology of acute GVHD. Using RNA-Seq, Chakraverty's group reported that effector $\mathrm{T}$ cells were reprogrammed by tissue-autonomous mechanisms in target organs for site-specific proinflammatory functions that were highly divergent from those primed in lymph nodes (107). New tools will likely soon provide major information about processes occurring in situ in the GVHD target organs. This includes tissue analyses using mass cytometry that extend labeling to over 40 markers (127) or combined single-cell transcriptomics with labeled antibodies (CITE-Seq; ref. 128).

Finally, an ongoing next step is to integrate multi-omics results with basic clinical characteristics that influence the incidence and the severity of GVHD. This represents the new vanguard in our quest to understand and control GVHD. 


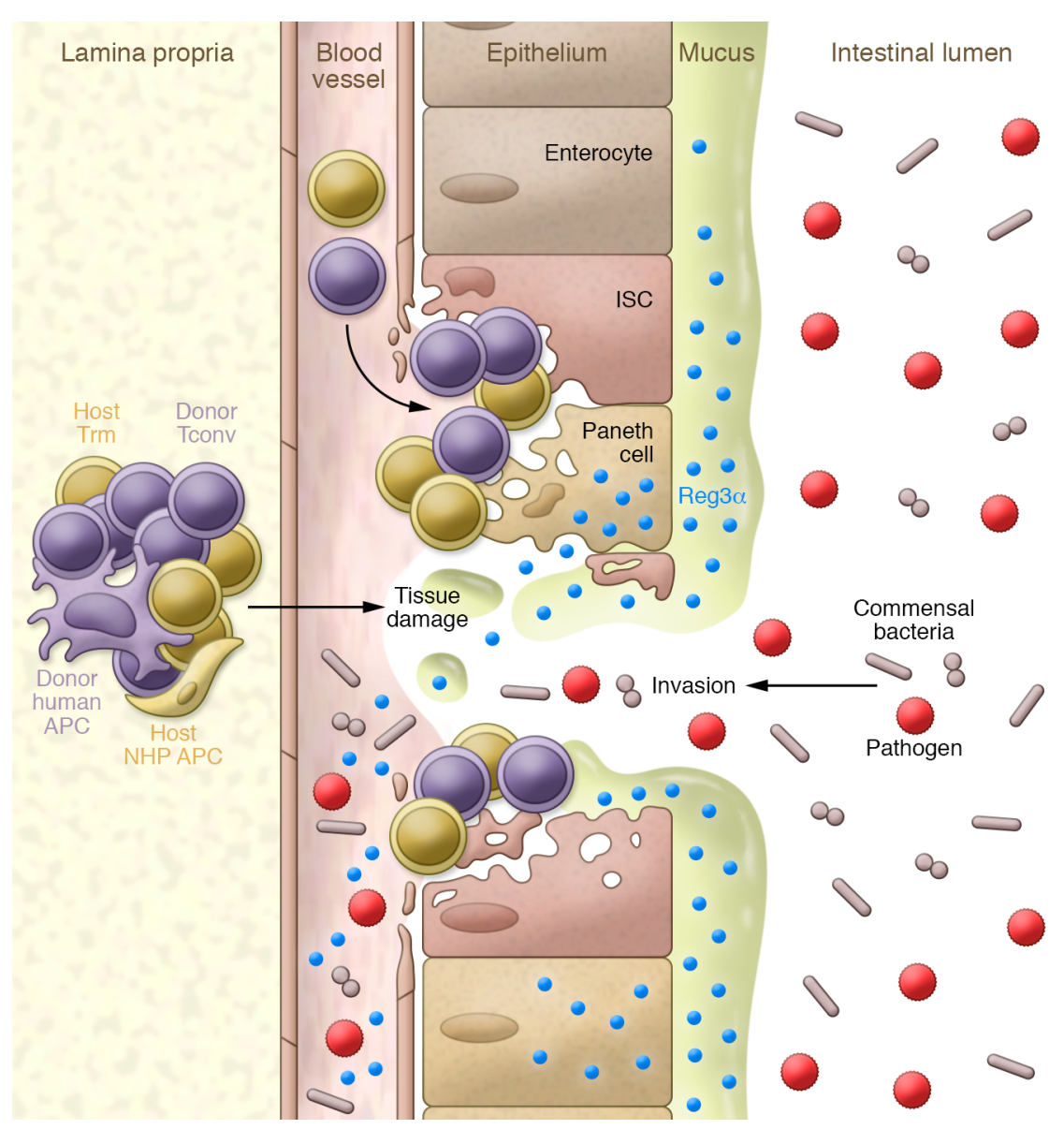

Figure 3. Emerging role of host Trms in GVHD pathophysiology. This figure illustrates the emerging role of Trms in CVHD pathology and the unexpected role of host-derived Trms in pathology through a host-versus-graft reaction; recent data suggest that host Trms in patient target organs can contribute to tissue destruction. ISC, intestinal stem cell; APC, antigen-presenting cell; NHP, nonhuman primate.

\section{Conclusions}

For years, translation of pathophysiologic insights to clinical management has relied on experimental findings (Figure 1). Despite progress and some successes in the preclinical-to-clinical translation of therapeutics and improved simulation of GVHD immunobiological mechanisms, some detractors claim such an approach has failed and should be abandoned. However, we would like to argue three points: (a) To date, preclinical modeling has led to several advancements in the translation of immunologic mechanisms into the clinic, resulting in new prophylactic and therapeutic interventions. (b) As new data become available, continued readjustments in accepted pathophysiologic paradigms and approaches are needed. (c) Even with the most promising potential therapeutics identified through preclinical experiments, development can be hampered both by inaccurate clinical/statistical assumptions of clinical response or survival rates, and by patient populations that are heterogeneous and not sufficiently stratified for risk factors or trials not statistically powered to test outcomes. Strengths and weaknesses of experimental models and of clinical trials are summarized in Table 1.

Here, we would like to advocate for the concept of the yin and yang approach to translational research: as a pendulum from mice/NHPs to the clinic, and then back to the lab (and vice versa: from translational medicine to clinical trials and back to the lab; Figure 2).
Finally, the rapid evolution of our understanding of the mechanistic drivers of GVHD reminds us that we should always be extremely careful with dogma. For years, based on peripheral blood studies, we believed that conditioning and GVHD eliminated most (if not all) of the recipients' hematopoietic cells. The recent discovery of potent host-derived tissue damage during GVHD (18, $95,96)$, and the still incomplete understanding of Trm dynamics (Figure 3), should again remind us that, as is the case in all scientific inquiry, the phrase "It's well known that" should be prohibited, or at least received with a healthy dose of circumspection.

\section{Acknowledgments}

The authors acknowledge support from a research grant by Alexion (to GS) and by Deutsche Krebshilfe (grant 70113473) and the José Carreras Leukaemia Foundation (grant DJCLS 01R/2019) (to RZ); and NIH grants R37 AI34495, 2 R01 HL56067, R01 HL11879, R01 HL155114, R01 AI056299, and P01 CA142106 (to BRB).

Address correspondence to: Gérard Socié, Hematology-Transplantation, Hospital Saint Louis, 1 Avenue Claude Vellefaux, 75010 Paris, France. Email: gerard.socie@aphp.fr. Or to: Bruce Blazar, Department of Pediatrics, Division of Pediatric Blood and Marrow Transplantation \& Cellular Therapy, MMC 366, 420 Delaware Street SE, Minneapolis, Minnesota, USA. Email: blaza001@umn.edu. 
1. Ferrara JLM, et al. Graft-versus-host disease. Lancet Lond Engl. 2009;373(9674):1550-1561.

2. Filipovich AH, et al. National Institutes of Health consensus development project on criteria for clinical trials in chronic graft-versus-host disease: I. Diagnosis and staging working group report. Biol Blood Marrow Transplant. 2005;11(12):945-956.

3. Jagasia MH, et al. National Institutes of Health consensus development project on criteria for clinical trials in chronic graft-versus-host disease: I. The 2014 Diagnosis and Staging Working Group report. Biol Blood Marrow Transplant. 2015;21(3):389-401.

4. Zeiser R, Blazar BR. Acute graft-versus-host disease - biologic process, prevention, and therapy. N Engl JMed. 2017;377(22):2167-2179.

5. Zeiser R, Blazar BR. Pathophysiology of chronic graft-versus-host disease and therapeutic targets. N Engl JMed. 2017;377(26):2565-2579.

6. Hill GR, et al. Current concepts and advances in graft-versus-host disease immunology. Annu Rev Immunol. 2021;39:19-49.

7. Socié G, Ritz J. Current issues in chronic graftversus-host disease. Blood. 2014;124(3):374-384.

8. Storb R, et al. Marrow grafts between canine litter-mates homozygous or heterozygous for lymphocyte-defined histocompatibility antigens. Transplantation. 1976;21(4):299-306.

9. Kolb H, et al. Pathology of acute graft-versus-host disease in the dog. An autopsy study of ninety-five dogs. Am J Pathol. 1979;96(2):581-594.

10. Atkinson K, et al. Acute and chronic graft-versushost disease in dogs given hemopoietic grafts from DLA-nonidentical littermates. Two distinct syndromes. Am J Pathol. 1982;108(2):196-205.

11. Graves SS, et al. A canine model of chronic graftversus-host disease. Biol Blood Marrow Transplant. 2017;23(3):420-427.

12. Burwitz BJ, et al. Allogeneic stem cell transplantation in fully MHC-matched Mauritian cynomolgus macaques recapitulates diverse human clinical outcomes. Nat Commun. 2017;8(1):1418.

13. Miller WP, et al. GVHD after haploidentical transplantation: a novel, MHC-defined rhesus macaque model identifies CD28- ${ }^{-} 8^{+}{ }^{+} \mathrm{T}$ cells as a reservoir of breakthrough $\mathrm{T}$-cell proliferation during costimulation blockade and sirolimus-based immunosuppression. Blood. 2010;116(24):5403-5418.

14. Tkachev V, et al. Combined OX4OL and mTOR blockade controls effector $\mathrm{T}$ cell activation while preserving Treg reconstitution after transplant. Sci Transl Med. 2017;9(408):eaan3085.

15. Watkins BK, et al. CD28 blockade controls T cell activation to prevent graft-versus-host disease in primates. J Clin Invest. 2018;128(9):3991-4007.

16. Furlan SN, et al. Transcriptome analysis of GVHD reveals aurora kinase A as a targetable pathway for disease prevention. Sci Transl Med. 2015;7(315):315ra191.

17. Furlan SN, et al. Systems analysis uncovers inflammatory $\mathrm{Th} / \mathrm{Tc} 17$-driven modules during acute GVHD in monkey and human T cells. Blood. 2016;128(21):2568-2579.

18. Tkachev V, et al. Spatiotemporal single-cell profiling reveals that invasive and tissue-resident memory donor $\mathrm{CD}^{+} \mathrm{T}$ cells drive gastrointestinal acute graft-versus-host disease. Sci Transl
Med. 2021;13(576):eabc0227.

19. Latis E, et al. Cellular and molecular profiling of T-cell subsets at the onset of human acute GVHD. Blood Adv. 2020;4(16):3927-3942.

20. Watkins B, et al. Phase II trial of costimulation blockade with abatacept for prevention of acute GVHD [published online January 15, 2021]. JClin Oncol. https://doi.org/10.1200/JCO.20.01086.

21. Morris H, et al. Tracking donor-reactive $\mathrm{T}$ cells: evidence for clonal deletion in tolerant kidney transplant patients. Sci Transl Med. 2015;7(272):272ra10.

22. Zuber J, et al. Bidirectional intragraft alloreactivity drives the repopulation of human intestinal allografts and correlates with clinical outcome. Sci Immunol. 2016;1(4):eaah3732.

23. Davis MM, Brodin P. Rebooting human immunology. Annu Rev Immunol. 2018;36:843-864.

24. Pulendran B, Davis MM. The science and medicine of human immunology. Science. 2020;369(6511):eaay4014.

25. Koyama M, et al. MHC class II antigen presentation by the intestinal epithelium initiates graft-versus-host disease and is influenced by the microbiota. Immunity. 2019;51(5):885-898.

26. Peled JU, et al. Microbiota as predictor of mortality in allogeneic hematopoietic-cell transplantation. N Engl JMed. 2020;382(9):822-834.

27. Schott C, et al. Bronchiolitis obliterans syndrome susceptibility and the pulmonary microbiome. J Heart Lung Transplant. 2018;37(9):1131-1140.

28. Koyama M, Hill GR. The primacy of gastrointestinal tract antigen-presenting cells in lethal graft-versus-host disease. Blood. 2019;134(24):2139-2148.

29. Fu YY, et al. T cell recruitment to the intestinal stem cell compartment drives immune-mediated intestinal damage after allogeneic transplantation. Immunity. 2019;51(1):90-103.

30. Takashima S, et al. T cell-derived interferon- $\gamma$ programs stem cell death in immune-mediated intestinal damage. Sci Immunol. 2019;4(42):eaay8556.

31. Matsuzawa-Ishimoto $Y$, et al. An intestinal organoid-based platform that recreates susceptibility to T-cell-mediated tissue injury. Blood. 2020;135(26):2388-2401.

32. Zeiser R, et al. Ruxolitinib for glucocorticoid-refractory acute graft-versus-host disease. $\mathrm{N} \mathrm{Engl} \mathrm{J}$ Med. 2020;382(19):1800-1810.

33. Zeiser R, Socié G. The development of ruxolitinib for glucocorticoid-refractory acute graft-versushost disease. Blood Adv. 2020;4(15):3789-3794.

34. Spoerl S, et al. Activity of therapeutic JAK $1 / 2$ blockade in graft-versus-host disease. Blood. 2014;123(24):3832-3842.

35. Stickel N, et al. MicroRNA-146a reduces MHC-II expression via targeting JAK/STAT signaling in dendritic cells after stem cell transplantation. Leukemia. 2017;31(12):2732-2741.

36. Hülsdünker J, et al. Neutrophils provide cellular communication between ileum and mesenteric lymph nodes at graft-versus-host disease onset. Blood. 2018;131(16):1858-1869.

37. Zeiser R, et al. Ruxolitinib in corticosteroid-refractory graft-versus-host disease after allogeneic stem cell transplantation: a multicenter survey. Leukemia. 2015;29(10):2062-2068.

38. Jagasia M, et al. Ruxolitinib for the treatment of steroid-refractory acute GVHD (REACH1): a multicenter, open-label phase 2 trial. Blood. 2020;135(20):1739-1749.

39. Zeiser R, et al. Ruxolitinib (RUX) vs best available therapy (BAT) in patients with steroid-refractory/steroid-dependent chronic graft-vs-host disease (cGVHD): primary findings from the phase 3, randomized REACH3 Study. Blood. 2020;136(suppl 1):22-24.

40. Choi J, et al. Baricitinib-induced blockade of interferon gamma receptor and interleukin-6 receptor for the prevention and treatment of graft-versus-host disease. Leukemia. 2018;32(11):2483-2494.

41. Schroeder MA, et al. A phase 1 trial of itacitinib, a selective JAK1 inhibitor, in patients with acute graft-versus-host disease. Blood Adv. 2020;4(8):1656-1669.

42. Zeiser R, et al. GRAVITAS-301: a randomized double-blinded phase 3 study of Itacitinib or placebo in combination with corticosteroids for initial treatment of patients with acute graft-versushost disease. EHA Library. 2020;295076:S256.

43. Blazar BR, et al. In vivo blockade of CD28/ CTLA4: B7/BB1 interaction with CTLA4-Ig reduces lethal murine graft-versus-host disease across the major histocompatibility complex barrier in mice. Blood. 1994;83(12):3815-3825.

44. Yu C, et al. Cytotoxic T lymphocyte antigen 4-immunoglobulin fusion protein combined with methotrexate/cyclosporine as graft-versus-host disease prevention in a canine dog leukocyte antigen-nonidentical marrow transplant model. Transplantation. 2000;69(3):450-454.

45. Koura DT, et al. In vivo T cell costimulation blockade with abatacept for acute graft-versus-host disease prevention: a first-in-disease trial. Biol Blood Marrow Transplant. 2013;19(11):1638-1649.

46. Blazar BR, et al. Blockade of CD40 ligandCD40 interaction impairs $\mathrm{CD}^{+}{ }^{+} \mathrm{T}$ cell-mediated alloreactivity by inhibiting mature donor $\mathrm{T}$ cell expansion and function after bone marrow transplantation. J Immunol. 1997;158(1):29-39.

47. Durham MM, et al. Cutting edge: administration of anti-CD40 ligand and donor bone marrow leads to hemopoietic chimerism and donor-specific tolerance without cytoreductive conditioning. JImmunol. 2000;165(1):1-4.

48. Kim SC, et al. Fc-silent anti-CD154 domain antibody effectively prevents nonhuman primate renal allograft rejection. Am J Transplant. 2017;17(5):1182-1192.

49. Blazar BR, et al. Ligation of OX40 (CD134) regulates graft-versus-host disease (GVHD) and graft rejection in allogeneic bone marrow transplant recipients. Blood. 2003;101(9):3741-3748.

50. Taylor PA, et al. Targeting of inducible costimulator (ICOS) expressed on alloreactive T cells down-regulates graft-versus-host disease (GVHD) and facilitates engraftment of allogeneic bone marrow (BM). Blood. 2005;105(8):3372-3380.

51. Pinelli DF, et al. An anti-CD154 domain antibody prolongs graft survival and induces Foxp3(+) iTreg in the absence and presence of CTLA-4 Ig. Am J Transplant. 2013;13(11):3021-3030.

52. Adom D, et al. $\mathrm{ICOSL}^{+}$plasmacytoid dendritic cells as inducer of graft-versus-host disease, responsive to a dual ICOS/CD28 antagonist. Sci Transl Med. 2020;12(564):eaay4799. 
53. Sato M, et al. Inducible costimulator (ICOS) up-regulation on activated $\mathrm{T}$ cells in chronic graft-versus-host disease after dog leukocyte antigen-nonidentical hematopoietic cell transplantation: a potential therapeutic target. Trans plantation. 2013;96(1):34-41

54. Kean LS, et al. Advances in targeting co-inhibitory and co-stimulatory pathways in transplantation settings: the Yin to the Yang of cancer immunotherapy. Immunol Rev. 2017;276(1):192-212.

55. Chung J, et al. Early notch signals induce a pathogenic molecular signature during priming of alloantigen-specific conventional $\mathrm{CD} 4^{+} \mathrm{T}$ cells in graft-versus-host disease. Jimmunol. 2019;203(2):557-568.

56 . Radojcic $V$, et al. Notch signaling mediated by Delta-like ligands 1 and 4 controls the pathogenesis of chronic GVHD in mice. Blood. 2018;132(20):2188-2200.

57. Chung J, et al. Fibroblastic niches prime T cell alloimmunity through Delta-like Notch ligands. J Clin Invest. 2017;127(4):1574-1588.

58. Rafei H, Jenq RR. Microbiome-intestine cross talk during acute graft-versus-host disease. Blood. 2020;136(4):401-409.

59. Jenq RR, et al. Regulation of intestinal inflammation by microbiota following allogeneic bone marrow transplantation. J Exp Med. 2012;209(5):903-911.

60. Taur Y, et al. The effects of intestinal tract bacterial diversity on mortality following allogeneic hematopoietic stem cell transplantation. Blood. 2014;124(7):1174-1182.

61. Weber D, et al. Rifaximin preserves intestinal microbiota balance in patients undergoing allogeneic stem cell transplantation. Bone Marrow Transplant. 2016;51(8):1087-1092.

62. Jenq RR, et al. Intestinal blautia is associated with reduced death from graft-versus-host disease. Biol Blood Marrow Transplant. 2015;21(8):1373-1383.

63. Stein-Thoeringer CK, et al. Lactose drives Enterococcus expansion to promote graft-versushost disease. Science. 2019;366(6469):1143-1149.

64. Mathewson ND, et al. Gut microbiome-derived metabolites modulate intestinal epithelial cell damage and mitigate graft-versus-host disease. Nat Immunol. 2016;17(5):505-513.

65. Shono Y, et al. Increased GVHD-related mortality with broad-spectrum antibiotic use after allogeneic hematopoietic stem cell transplantation in human patients and mice. Sci Transl Med. 2016;8(339):339ra71.

66. Hülsdünker J, et al. Immunization against poly-N-acetylglucosamine reduces neutrophil activation and GVHD while sparing microbial diversity. Proc Natl Acad Sci U S A. 2019;116(41):20700-20706

67. Legoff J, et al. The eukaryotic gut virome in hematopoietic stem cell transplantation: new clues in enteric graft-versus-host disease. Nat Med. 2017;23(9):1080-1085.

68. Zhang $\mathrm{F}$, et al. Longitudinal dynamics of gut bacteriome, mycobiome and virome after fecal microbiota transplantation in graft-versus-host disease. Nat Commun. 2021;12(1):65.

69. Kakihana K, et al. Fecal microbiota transplantation for patients with steroid-resistant acute graft-versus-host disease of the gut. Blood.
2016;128(16):2083-2088.

70. DeFilipp Z, et al. Third-party fecal microbiota transplantation following allo-HCT reconstitutes microbiome diversity. Blood Adv. 2018;2(7):745-753.

71. van Lier YF, et al. Donor fecal microbiota transplantation ameliorates intestinal graftversus-host disease in allogeneic hematopoietic cell transplant recipients. Sci Transl Med. 2020;12(556):eaaz8926.

72. Schwab L, et al. Neutrophil granulocytes recruited upon translocation of intestinal bacteria enhance graft-versus-host disease via tissue damage. Nat Med.2014;20(6):648-654.

73. Bruce DW, et al. Type 2 innate lymphoid cells treat and prevent acute gastrointestinal graft-versushost disease. JClin Invest. 2017;127(5):1813-1825.

74. Holtan SG, et al. Amphiregulin modifies the Minnesota Acute Graft-versus-Host Disease Risk Score: results from BMT CTN 0302/0802. Blood Adv. 2018;2(15):1882-1888.

75. Michonneau D, et al. Metabolomics analysis of human acute graft-versus-host disease reveals changes in host and microbiota-derived metabolites. Nat Commun. 2019;10(1):5695.

76. Haring $\mathrm{E}$, et al. Bile acids regulate intestinal antigen presentation and reduce graft-versus-host disease without impairing the graft-versus-leukemia effect [published online July 16, 2020]. Haematologica. https://doi.org/10.3324/haematol.2019.242990.

77. Brown RA, Byersdorfer CA. Metabolic pathways in alloreactive T cells. Front Immunol. 2020;11:1517.

78. Uhl FM, et al. Metabolic reprogramming of donor $\mathrm{T}$ cells enhances graft-versus-leukemia effects in mice and humans. Sci Transl Med. 2020;12(567):eabb8969.

79. Assmann JC, et al. Glycolytic metabolism of pathogenic T cells enables early detection of GVHD by 13C-MRI. Blood. 2021;137(1):126-137.

80. Nguyen HD, et al. Lysosomal acid lipase is required for donor $\mathrm{T}$ cells to induce graft-versushost disease. Cell Rep. 2020;33(4):108316.

81. Nguyen HD, et al. Metabolic reprogramming of alloantigen-activated T cells after hematopoietic cell transplantation. J Clin Invest. 2016;126(4):1337-1352.

82. Wu SR, Reddy P. Tissue tolerance: a distinct concept to control acute GVHD severity. Blood. 2017;129(13):1747-1752.

83. Panoskaltsis-Mortari A, et al. Keratinocyte growth factor administered before conditioning ameliorates graft-versus-host disease after allogeneic bone marrow transplantation in mice. Blood.1998;92(10):3960-3967.

84. Spielberger R, et al. Palifermin for oral mucositis after intensive therapy for hematologic cancers. N Engl J Med. 2004;351(25):2590-2598.

85. Blazar BR, et al. Phase $1 / 2$ randomized, placebocontrol trial of palifermin to prevent graft-versushost disease (GVHD) after allogeneic hematopoietic stem cell transplantation (HSCT). Blood. 2006;108(9):3216-3222.

86. Keir M, et al. The role of IL-22 in intestinal health and disease. JExp Med. 2020;217(3):e20192195.

87. Lindemans CA, et al. Interleukin-22 promotes intestinal-stem-cell-mediated epithelial regeneration. Nature. 2015;528(7583):560-564.

88. Takashima S, et al. The Wnt agonist R-spondin 1 regulates systemic graft-versus-host disease by protecting intestinal stem cells. J Exp Med. 2011;208(2):285-294.

89. Hayase E, et al. R-Spondin1 expands Paneth cells and prevents dysbiosis induced by graft-versushost disease. J Exp Med. 2017;214(12):3507-3518

90. Norona J, et al. Glucagon-like peptide 2 for intestinal stem cell and Paneth cell repair during graftversus-host disease in mice and humans. Blood. 2020;136(12):1442-1455.

91. Rosato PC, et al. Integrating resident memory into T cell differentiation models. Curr Opin Immunol. 2020;63:35-42.

92. Masopust D, Soerens AG. Tissue-resident T cells and other resident leukocytes. Annu Rev Immunol. 2019;37:521-546.

93. El-Asady R, et al. TGF- $\beta$-dependent CD103 expression by CD8(+) T cells promotes selective destruction of the host intestinal epithelium during graft-versus-host disease. JExp Med. 2005;201(10):1647-1657.

94. Morin-Zorman S, et al. In vivo dynamics of $\mathrm{T}$ cells and their interactions with dendritic cells in mouse cutaneous graft-versus-host disease. Blood Adv. 2019;3(14):2082-2092.

95. Strobl J, et al. Long-term skin-resident memory $T$ cells proliferate in situ and are involved in human graft-versus-host disease. Sci Transl Med. 2020;12(570):eabb7028.

96. Divito SJ, et al. Peripheral host T cells survive hematopoietic stem cell transplantation and promote graft-versus-host disease. JClin Invest. 2020;130(9):4624-4636.

97. Radojcic V, Luznik L. Mechanism of action of posttransplantation cyclophosphamide: more than meets the eye. J Clin Invest. 2019;129(6):2189-2191.

98. Luznik L, et al. High-dose cyclophosphamide as single-agent, short-course prophylaxis of graft-versus-host disease. Blood. 2010;115(16):3224-3230.

99. Ganguly S, et al. Donor $\mathrm{CD}^{+}{ }^{+}$Foxp $^{+}$regulatory $\mathrm{T}$ cells are necessary for posttransplantation cyclophosphamide-mediated protection against GVHD in mice. Blood. 2014;124(13):2131-2141.

100.Kanakry CG, et al. Aldehyde dehydrogenase expression drives human regulatory $\mathrm{T}$ cell resistance to posttransplantation cyclophosphamide. Sci Transl Med. 2013;5(211):211ra157.

101. Wachsmuth LP, et al. Posttransplantation cyclophosphamide prevents graft-versus-host disease by inducing alloreactive $\mathrm{T}$ cell dysfunction and suppression. JClin Invest. 2019;129(6):2357-2373.

102. Cooke KR, et al. The biology of chronic graftversus-host disease: a task force report from the National Institutes of Health Consensus Development Project on Criteria for Clinical Trials in Chronic Graft-versus-Host Disease. Biol Blood Marrow Transplant. 2017;23(2):211-234.

103. MacDonald KP, et al. Cytokine mediators of chronic graft-versus-host disease. J Clin Invest. 2017;127(7):2452-2463.

104. Dudakov JA, et al. Loss of thymic innate lymphoid cells leads to impaired thymopoiesis in experimental graft-versus-host disease. Blood. 2017;130(7):933-942.

105. Du J, et al. Pirfenidone ameliorates murine chronic GVHD through inhibition of macrophage infiltration and TGF- $\beta$ production. Blood. 2017;129(18):2570-2580. 
106. Du J, et al. Invariant natural killer $\mathrm{T}$ cells ameliorate murine chronic GVHD by expanding donor regulatory T cells. Blood. 2017;129(23):3121-3125.

107. Dertschnig S, et al. Graft-versus-host disease reduces lymph node display of tissue-restricted self-antigens and promotes autoimmunity. JClin Invest. 2020;130(4):1896-1911.

108. Lerbs T, et al. CD47 prevents the elimination of diseased fibroblasts in scleroderma. JCI Insight. 2020;5(16):e140458.

109. Forcade E, et al. An activated Th17-prone T cell subset involved in chronic graft-versus-host disease sensitive to pharmacological inhibition. JCI Insight. 2017;2(12):e92111.

110. Flynn R, et al. Targeted Rho-associated kinase 2 inhibition suppresses murine and human chronic GVHD through a Stat3-dependent mechanism. Blood.2016;127(17):2144-2154.

111. Paz K, et al. Small-molecule BCL6 inhibitor effectively treats mice with nonsclerodermatous chronic graft-versus-host disease. Blood. 2019;133(1):94-99.

112. Verghese DA, et al. C5aR1 regulates T follicular helper differentiation and chronic graft-versushost disease bronchiolitis obliterans. JCI Insight. 2018;3(24):e124646.

113. Kong $\mathrm{X}$, et al. Tissue-resident PSGL1 $1^{10} \mathrm{CD} 4{ }^{+}$ $\mathrm{T}$ cells promote $\mathrm{B}$ cell differentiation and chronic graft-versus-host disease-associated autoimmunity. J Clin Invest. 2021;131(1):e135468.

114. Srinivasan M, et al. Donor B-cell alloantibody deposition and germinal center formation are required for the development of murine chronic GVHD and bronchiolitis obliterans. Blood. 2012;119(6):1570-1580.

115. Kolupaev OV, et al. Impaired bone marrow B-cell development in mice with a bronchiolitis obliterans model of cGVHD. Blood Adv . 2018;2(18):2307-2319.

116. Allen JL, et al. Increased BCR responsiveness in B cells from patients with chronic GVHD. Blood. 2014;123(13):2108-2115.

117. Jia $\mathrm{W}$, et al. BAFF promotes heightened BCR responsiveness and manifestations of chronic GVHD after allogeneic stem cell transplantation. Blood. 2021;137(18):2544-2557.

118. Flynn R, et al. Targeting Syk-activated B cells in murine and human chronic graft-versus-host disease. Blood. 2015;125(26):4085-4094.

119. Poe JC, et al. SYK inhibitor entospletinib prevents ocular and skin GVHD in mice. JCI Insight. 2018;3(19):e122430.

120. Dubovsky JA, et al. Ibrutinib treatment ameliorates murine chronic graft-versus-host disease. JClin Invest. 2014;124(11):4867-4876.

121. Paz K, et al. Targeting PI3K $\delta$ function for ame- lioration of murine chronic graft-versus-host disease. Am J Transplant. 2019;19(6):1820-1830.

122. Thangavelu $\mathrm{G}$, et al. Inhibition of inositol kinase $B$ controls acute and chronic graft-versus-host disease. Blood. 2020;135(1):28-40.

123. Allen JL, et al. B cells from patients with chronic GVHD are activated and primed for survival via BAFF-mediated pathways. Blood. 2012;120(12):2529-2536.

124.Jacobson CA, et al. Post-transplantation B cell activating factor and $\mathrm{B}$ cell recovery before onset of chronic graft-versus-host disease. Biol Blood Marrow Transplant. 2014;20(5):668-675.

125. Lakshmikanth T, et al. Mass cytometry and topological data analysis reveal immune parameters associated with complications after allogeneic stem cell transplantation. Cell Rep. 2017;20(9):2238-2250.

126.Jardine L, et al. Donor monocyte-derived macrophages promote human acute graft-versus-host disease. J Clin Invest. 2020;130(9):4574-4586.

127. Keren L, et al. A structured tumor-immune microenvironment in triple negative breast cancer revealed by multiplexed ion beam imaging. Cell. 2018;174(6):1373-1387.

128.Stoeckius M, et al. Simultaneous epitope and transcriptome measurement in single cells. Nat Methods. 2017;14(9):865-868. 